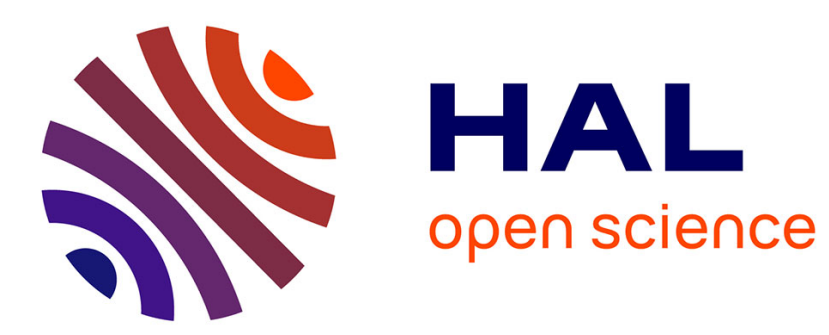

\title{
Response of periodic structures due to moving loads
} Hamid Chebli, Ramzi Othman, Didier Clouteau

\section{To cite this version:}

Hamid Chebli, Ramzi Othman, Didier Clouteau. Response of periodic structures due to moving loads. Comptes Rendus Mécanique, 2006, 334, pp.347-352. 10.1016/j.crme.2006.04.001 . hal-01007256

\section{HAL Id: hal-01007256 https://hal.science/hal-01007256}

Submitted on 3 Jun 2017

HAL is a multi-disciplinary open access archive for the deposit and dissemination of scientific research documents, whether they are published or not. The documents may come from teaching and research institutions in France or abroad, or from public or private research centers.
L'archive ouverte pluridisciplinaire HAL, est destinée au dépôt et à la diffusion de documents scientifiques de niveau recherche, publiés ou non, émanant des établissements d'enseignement et de recherche français ou étrangers, des laboratoires publics ou privés.

\section{(c)(1)}

Distributed under a Creative Commons Attribution| 4.0 International License 


\title{
Response of periodic structures due to moving loads
}

\author{
Hamid Chebli, Ramzi Othman, Didier Clouteau \\ LMSSMat, École centrale Paris, grande voie des vignes, 92295 Châtenay-Malabry cedex, France
}

\begin{abstract}
A method is proposed to calculate the response of periodic structures subjected to moving loads. It is based on the Floquet decomposition which allows the restriction of the analysis for the overall system to a generic cell. The main contribution of the approach presented hereafter is that the response is directly deduced from transfer functions in the space-wavenumber domain calculated in an unbounded generic cell. Moreover, the equivalence of this new solution with the response of invariant structures obtained using Fourier transforms is established.
\end{abstract}

\section{Résumé}

Réponse de structures périodiques à des charges mobiles. Une méthode est proposée afin de calculer la réponse de structures périodiques soumises à des charges mobiles. Celle-ci est basée sur la décomposition de Floquet qui permet de restreindre l'analyse du système entier à une cellule de référence. La principale contribution de l'approche présentée ci-après est que la réponse est directement déduite à partir de la fonction de transfert dans le domaine espace-nombre d'onde calculée dans une cellule de référence non bornée. De plus, l'équivalence entre la solution obtenue et la réponse de structures invariantes calculée avec la transformée de Fourier est établie.

Keywords: Solids and structures; Periodic structure; Floquet transform; Moving load

Mots-clés : Solides et structures ; Structure périodique; Transformée de Floquet ; Charge mobile

\section{Introduction}

Vibrations induced by railway traffic are of a major concern [1] since they have an important impact on the human comfort and on the built environment. The study of these vibrations requires three-dimensional (3D) models of unbounded domains, that is the soil-railway track.

In this Note, these domains are supposed to be periodic along one given direction. Then, the analysis for the overall system is substituted by one for a generic cell using the Floquet decomposition [2]. This paradigm has been already 
applied, but only to $1 \mathrm{D}$ or 2D periodically supported structures [3,4] for which the generic cell is then bounded. In order to take into account a structure interacting with a soil half-space, a new method [5] has been proposed which allows for 3D unbounded generic cells. This approach has been initially developed for the study of the dynamic responses of structures subjected to 3D seismic loading. This methodology is here extended to the case of moving loads and a new formulation is presented in which the response is directly deduced from transfer functions calculated in the unbounded generic cell.

In a first part, the Floquet transform is defined. Its relation with the Fourier transform is introduced: this formula is used in the last paragraph to get the relationship between the responses due to moving loads of periodic and invariant structures. In Section 3, the generic problem is built. It corresponds to the dynamic problem posed in the reference cell. Section 4 is devoted to the expression of the response of periodic structures due to moving loads. The proposed method is then applied for a real railway structure. Finally, the connection with the case of invariant structures is presented in Section 5.

\section{Floquet transforms}

In this section, we recall the important results related to Floquet transforms. For a deeper review, one can refer to [2]. Moreover, relations between Floquet and Fourier transforms are introduced.

\subsection{Definitions}

Let $\Omega$ be an unbounded open set. The position vector of any point of this domain is given by:

$$
\mathbf{x}=x_{1} \mathbf{e}_{1}+x_{2} \mathbf{e}_{2}+x_{3} \mathbf{e}_{3}
$$

with $\left(\mathbf{e}_{1}, \mathbf{e}_{2}, \mathbf{e}_{3}\right)$ a Cartesian reference system. The domain $\Omega$ is assumed to be periodic in the $\mathbf{e}_{2}$ direction with a period $L$, i.e. it is invariant through any translation of vector $n L \mathbf{e}_{2}$, where $n$ is a signed integer. The Floquet transform of any function $f$ defined in $\Omega$ is a function $\tilde{f}$ defined in $\widetilde{\Omega} \times[-\pi / L, \pi / L]$, with $\widetilde{\Omega}=\left\{\mathbf{x} \in \Omega \mid 0<\mathbf{x} . \mathbf{e}_{2}<L\right\}$, and such that:

$$
\tilde{f}(\tilde{\mathbf{x}}, \kappa)=\sum_{n=-\infty}^{+\infty} f\left(\tilde{\mathbf{x}}+n L \mathbf{e}_{2}\right) \mathrm{e}^{\mathrm{i} n \kappa L}
$$

in which the wavenumber $\kappa \in[-\pi / L, \pi / L]$ and where $\tilde{\mathbf{x}}$ is the position vector in the reference cell $\widetilde{\Omega}$ defined by $\tilde{\mathbf{x}}=\tilde{x}_{1} \mathbf{e}_{1}+\tilde{x}_{2} \mathbf{e}_{2}+\tilde{x}_{3} \mathbf{e}_{3}$ with $\tilde{x}_{1}=x_{1}, \tilde{x}_{2}=x_{2}-n L, \tilde{x}_{3}=x_{3}$. It should be noted that the Floquet transform $\tilde{f}$ is periodic of the second kind, that is:

$$
\tilde{f}\left(\tilde{\mathbf{x}}+L \mathbf{e}_{2}, \kappa\right)=\mathrm{e}^{-\mathrm{i} \kappa L} \tilde{f}(\tilde{\mathbf{x}}, \kappa)
$$

Finally, for any $\mathbf{x}=\tilde{\mathbf{x}}+n L \mathbf{e}_{2}(\mathbf{x}$ in $\Omega$ and $\tilde{\mathbf{x}}$ in $\widetilde{\Omega}), f$ may be recovered from its Floquet transform by:

$$
f(\mathbf{x})=\frac{L}{2 \pi} \int_{0}^{2 \pi / L} \tilde{f}(\tilde{\mathbf{x}}, \kappa) \mathrm{e}^{-\mathrm{i} n \kappa L} \mathrm{~d} \kappa
$$

\subsection{Relations between Floquet and Fourier transforms}

Let $F(x)$ be a function (defined on $\mathbb{R}$ for the sake of simplicity). It is recalled that its Fourier transform $\hat{F}$ can be defined by:

$$
\hat{F}(k)=\int_{-\infty}^{+\infty} \mathrm{e}^{\mathrm{i} k x} F(x) \mathrm{d} x, \quad \forall k \in \mathbb{R}
$$

The function $F$ can be recovered from its Fourier transform as follows:

$$
F(x)=\frac{1}{2 \pi} \int_{-\infty}^{+\infty} \mathrm{e}^{-\mathrm{i} k x} \hat{F}(k) \mathrm{d} k
$$


Using these conventional formulae, the Fourier transform of the function $f_{x_{1}, x_{3}}$ defined by:

$$
f_{x_{1}, x_{3}}\left(x_{2}\right)=f(\mathbf{x})
$$

can be calculated from the Floquet transform of $f$ as follows [5]:

$$
\hat{f}_{x_{1}, x_{3}}\left(k_{x_{2}}\right)=\int_{0}^{L} \mathrm{e}^{\mathrm{i} k_{x_{2}} \tilde{x}_{2}} \tilde{f}\left(x_{1} \mathbf{e}_{1}+\tilde{x}_{2} \mathbf{e}_{2}+x_{3} \mathbf{e}_{3}, \kappa\right) \mathrm{d} \tilde{x}_{2}
$$

where $\kappa$ is in $[-\pi / L, \pi / L]$ and $k_{x_{2}}=\kappa+2 n \pi / L$. Moreover, the Floquet transform of $f$ is recovered from $\hat{f}_{x_{1}, x_{3}}$ by:

$$
\tilde{f}(\tilde{\mathbf{x}}, \kappa)=\frac{1}{2 \pi} \sum_{n=-\infty}^{+\infty} \mathrm{e}^{-\mathrm{i}\left(\kappa+\frac{2 n \pi}{L}\right) \tilde{x}_{2}} \hat{f}_{x_{1}, x_{3}}\left(\kappa+\frac{2 n \pi}{L}\right)
$$

\section{Generic problem associated to the periodic domain}

This section deals with the dynamic response of the soil-track system subjected to any external forces. This domain is assumed to have an elastic behaviour. Using Floquet transform defined previously, the dynamic problem for the overall system can be substituted by one posed in a reference cell. The generic problem is then introduced.

Let $\mathbf{u}(\mathbf{x}, t)$ be the elastodynamic displacement field in the unbounded domain $\Omega$. Its Fourier transform, $\hat{\mathbf{u}}(\mathbf{x}, \omega)$, satisfies:

$$
\begin{aligned}
& \operatorname{div} \sigma(\hat{\mathbf{u}})=-\rho \omega^{2} \hat{\mathbf{u}}, \quad \mathbf{x} \in \Omega \\
& \mathbf{t}(\hat{\mathbf{u}})=\mathbf{f}_{\mathbf{0}}, \quad \mathbf{x} \in \Gamma
\end{aligned}
$$

where $\rho$ is the mass density, $\sigma(\hat{\mathbf{u}})$ is the elastic stress tensor associated to the displacement field $\hat{\mathbf{u}}$ and $\mathbf{t}(\hat{\mathbf{u}})=\sigma(\hat{\mathbf{u}}) \mathbf{n}$ corresponds to the traction vector on the considered boundary using the outer normal convention for $\mathbf{n}$. Finally, $\Gamma$ is a part of the boundary of the domain $\Omega$ on which the external forces $\mathbf{f}_{\mathbf{0}}$ are applied.

Let $\check{\mathbf{u}}(\tilde{\mathbf{x}}, \kappa, \omega)$ be a displacement field that satisfies:

$$
\begin{aligned}
& \operatorname{div} \sigma(\check{\mathbf{u}})=-\rho \omega^{2} \check{\mathbf{u}}, \quad \tilde{\mathbf{x}} \in \widetilde{\Omega} \\
& \mathbf{t}(\check{\mathbf{u}})=\tilde{\mathbf{f}}_{\mathbf{0}}, \quad \tilde{\mathbf{x}} \in \widetilde{\Gamma} \\
& \check{\mathbf{u}}(\tilde{\mathbf{x}})=\mathrm{e}^{-\mathrm{i} \kappa L} \check{\mathbf{u}}\left(\tilde{\mathbf{x}}-L \mathbf{e}_{2}\right), \quad \tilde{\mathbf{x}} \in \widetilde{\Gamma}_{L}
\end{aligned}
$$

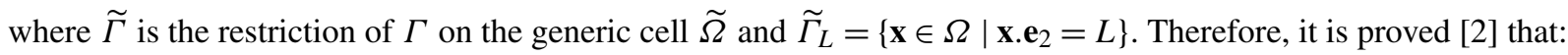

$$
\check{\mathbf{u}}=\tilde{\hat{\mathbf{u}}}
$$

where $\tilde{\hat{\mathbf{u}}}$ is the Floquet transform of $\hat{\mathbf{u}}$. Hence, instead of solving the problem for the whole domain (Eqs. (10) and (11)), one can solve the generic problem defined by Eqs. (12)-(14). The response in the overall domain is recovered from the response in the generic cell by using the inverse Floquet transform defined by Eq. (4). It should be noted here that a method [5] has been proposed to solve the generic problem in the case of an unbounded cell.

\section{Response of periodic structures due to moving loads}

In this section, the dynamic behaviour of the railway track structure is focused on. The external forces applying on it are supposed to be moving loads (which can model a train for instance).

Consider a general pinpoint load moving along the $\mathbf{e}_{2}$ axis with a constant velocity $V$ :

$$
\begin{aligned}
\mathbf{G}(\mathbf{y}, t) & =g\left(y_{2}-X_{2}\right) \delta\left(y_{1}-X_{1}\right) \delta\left(y_{2}-X_{2}-V t\right) \delta\left(y_{3}-X_{3}\right) \mathbf{e}_{3} \\
& =G\left(y_{2}, t\right) \delta\left(y_{1}-X_{1}\right) \delta\left(y_{3}-X_{3}\right) \mathbf{e}_{3}
\end{aligned}
$$

with $\mathbf{y}=y_{1} \mathbf{e}_{1}+y_{2} \mathbf{e}_{2}+y_{3} \mathbf{e}_{3}$ and where $\left(X_{1}, X_{2}, X_{3}\right)=\mathbf{X}$ is the position of the moving force at the time $t=0$; $G\left(y_{2}, t\right)=g\left(y_{2}-X_{2}\right) \delta\left(y_{2}-X_{2}-V t\right)$ in which $g\left(y_{2}-X_{2}\right)$ is the variation of the moving force amplitude. 
Without any lose of generality, we can assume that the point $\mathbf{x}$, where the response is calculated, is in the track generic cell. So $\mathbf{x}=\tilde{\mathbf{x}}$. Let $\hat{\mathbf{u}}(\mathbf{x}, \mathbf{X}, \omega)$ be the displacement in $\mathbf{x}$ due to a moving load acting on $\mathbf{X}$ at $t=0$. This field is given by:

$$
\hat{\mathbf{u}}(\mathbf{x}, \mathbf{X}, \omega)=\int_{-\infty}^{+\infty} \hat{G}\left(y_{2}, \omega\right) \hat{\mathbf{h}}\left(\mathbf{x}, \mathbf{y}=X_{1} \mathbf{e}_{1}+y_{2} \mathbf{e}_{2}+X_{3} \mathbf{e}_{3}, \omega\right) \mathrm{d} y_{2}
$$

where $\hat{\mathbf{h}}(\mathbf{x}, \mathbf{y}, \omega)$ is the displacement in the frequency domain of a point $\mathbf{x}$ due to an impulse force at a point $\mathbf{y}$. Moreover, $\hat{G}$ is the Fourier transform of $G$ and is given by:

$$
\hat{G}\left(y_{2}, \omega\right)=\mathrm{e}^{\mathrm{i} \frac{\omega}{V}\left(y_{2}-X_{2}\right)} g\left(y_{2}-X_{2}\right)
$$

For the sake of clarity, arguments $\mathbf{x}, \mathbf{X}$ and $\omega$ will be often omitted in the following. Then, replacing Eq. (18) in Eq. (17) and expressing $g\left(y_{2}-X_{2}\right)$ with its Fourier transform, one gets:

$$
\hat{\mathbf{u}}=\frac{1}{2 \pi} \int_{-\infty}^{+\infty} \int_{-\infty}^{+\infty} \mathrm{e}^{-\mathrm{i} k^{*}\left(y_{2}-X_{2}\right)} \hat{g}\left(k_{y_{2}}\right) \hat{\mathbf{h}}\left(y_{2}\right) \mathrm{d} y_{2} \mathrm{~d} k_{y_{2}}
$$

with $k^{*}=\left(k_{y_{2}}-\omega / V\right)$. Eq. (19) is also equivalent to:

$$
\hat{\mathbf{u}}=\frac{1}{2 \pi} \int_{-\infty}^{+\infty} \hat{g}\left(k_{y_{2}}\right) \sum_{n=-\infty}^{+\infty} \int_{n L}^{(n+1) L} \mathrm{e}^{-\mathrm{i} k^{*}\left(y_{2}-X_{2}\right)} \hat{\mathbf{h}}\left(y_{2}\right) \mathrm{d} y_{2} \mathrm{~d} k_{y_{2}}
$$

Taking $\tilde{y}_{2}=y_{2}-n L$ leads to:

$$
\hat{\mathbf{u}}=\frac{1}{2 \pi} \int_{-\infty}^{+\infty} \hat{g}\left(k_{y_{2}}\right) \int_{0}^{L} \sum_{n=-\infty}^{+\infty} \mathrm{e}^{-i k^{*}\left(\tilde{y}_{2}+n L-X_{2}\right)} \hat{\mathbf{h}}\left(\tilde{y}_{2}+n L\right) \mathrm{d} \tilde{y}_{2} \mathrm{~d} k_{y_{2}}
$$

Due to the geometric periodicity, we have:

$$
\hat{\mathbf{h}}\left(\mathbf{x}, \tilde{y}_{2}+n L\right)=\hat{\mathbf{h}}\left(\mathbf{x}-n L \mathbf{e}_{2}, \tilde{y}_{2}\right)
$$

Moreover, the Floquet transform of the function $\hat{\mathbf{h}}\left(\mathbf{x}, \tilde{y}_{2}\right)$, with respect to the variable $\mathbf{x}$, is the function $\tilde{\hat{\mathbf{h}}}_{\mathbf{x}}\left(\mathbf{x}, \tilde{y}_{2}, \kappa\right)$ defined by:

$$
\tilde{\hat{\mathbf{h}}}_{\mathbf{x}}\left(\mathbf{x}, \tilde{y}_{2}, \kappa\right)=\sum_{n=-\infty}^{+\infty} \hat{\mathbf{h}}\left(\mathbf{x}+n L \mathbf{e}_{2}, \tilde{y}_{2}\right) \mathrm{e}^{\mathrm{i} n \kappa L}
$$

From Eqs. (21)-(23) one gets:

$$
\hat{\mathbf{u}}(\mathbf{x})=\frac{1}{2 \pi} \int_{-\infty}^{+\infty} \hat{g}\left(k_{y_{2}}\right) \mathrm{e}^{\mathrm{i} k^{*} X_{2}} \int_{0}^{L} \mathrm{e}^{-\mathrm{i} k^{*} \tilde{y}_{2}} \tilde{\hat{\mathbf{h}}}_{\mathbf{x}}\left(\mathbf{x}, \tilde{y}_{2}, \kappa_{0}\right) \mathrm{d} \tilde{y}_{2} \mathrm{~d} k_{y_{2}}
$$

where $\kappa_{0}=k^{*}-\frac{2 m \pi}{L}, m$ being the signed integer such as $\left.\kappa_{0} \in\right]-\pi / L, \pi / L\left[\right.$. It is worth to notice that $\tilde{\hat{\mathbf{h}}}_{\mathbf{x}}$ is the solution of the problem (12)-(14) with $\tilde{\mathbf{f}}_{\mathbf{0}}(\tilde{\mathbf{x}})=\delta\left(\tilde{x}_{1}-X_{1}\right) \delta\left(\tilde{x}_{2}-\tilde{y}_{2}\right) \delta\left(\tilde{x}_{3}-X_{3}\right)$.

Eq. (24) has two advantages. At first, only the response of the generic cell is necessary instead of the response of the whole domain as needed in Eq. (17). Secondly, the displacement due to a moving load in the frequency domain is directly derived from the transfer function $\tilde{\hat{\mathbf{h}}}_{\mathbf{x}}$ in the $\omega-\kappa$ domain. Then, no inverse Floquet transform (see Eq. (4)) is needed.

In the case of a constant moving load, $G\left(y_{2}, t\right)$ defined by Eq. (16) is given by:

$$
G\left(y_{2}, t\right)=G_{0} \delta\left(y_{2}-X_{2}-V t\right)
$$




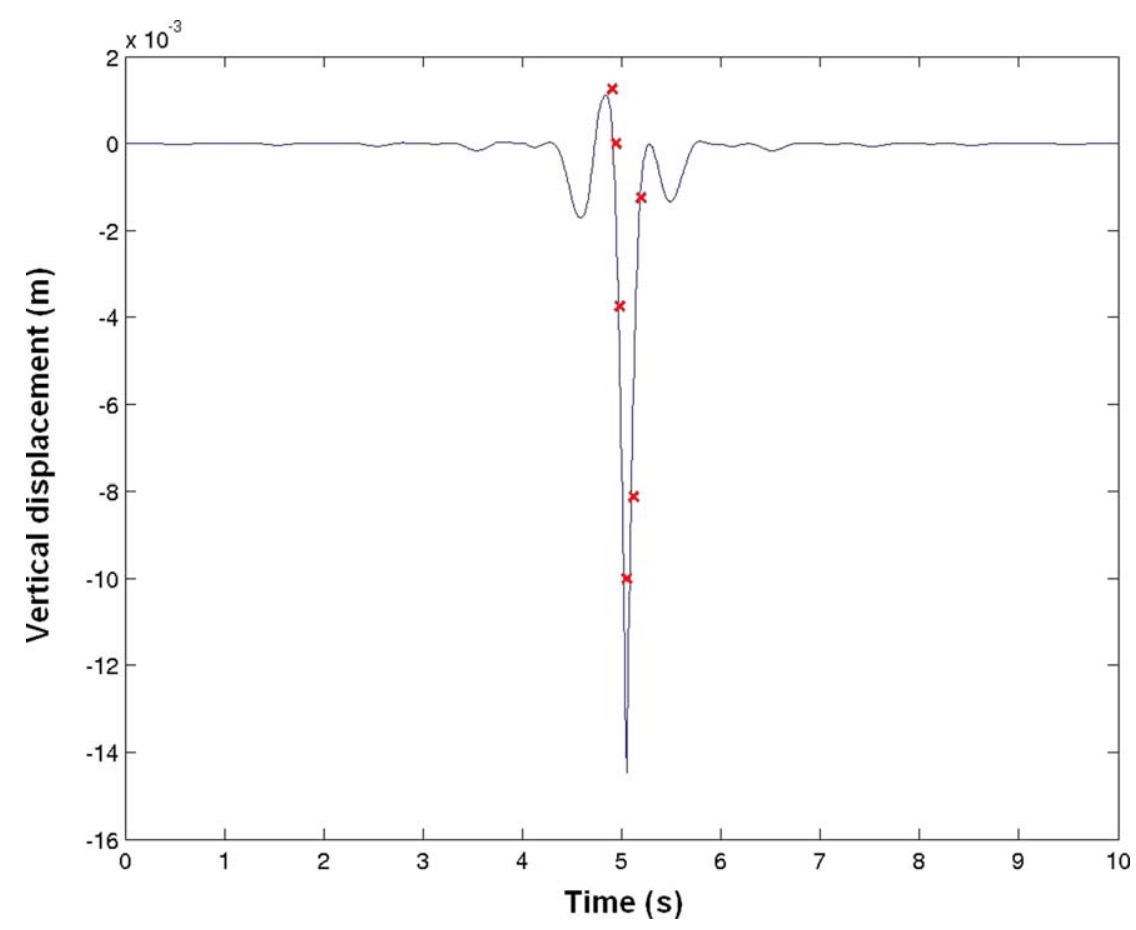

Fig. 1. Vertical displacement response with respect to time of a point in the railway track, due to the passage of one axle for a train travelling at $200 \mathrm{~km} / \mathrm{h}$. Comparison between measurement (crosses) and simulation (continuous line).

with $G_{0}$ a real constant. Eq. (24) is then equivalent to:

$$
\hat{\mathbf{u}}=\frac{G_{0}}{2 \pi} \mathrm{e}^{-\mathrm{i} \frac{\omega}{V} X_{2}} \int_{0}^{L} \mathrm{e}^{\mathrm{i} \frac{\omega}{V} \tilde{y}_{2}} \tilde{\hat{\mathbf{h}}}_{\mathbf{x}}\left(\tilde{y}_{2}, \kappa_{0}\right) \mathrm{d} \tilde{y_{2}}
$$

where $\kappa_{0}=-\frac{\omega}{V}-\frac{2 m \pi}{L}$. In this case, Fig. 1 compares simulations (continuous line) with measurements from [6] (cross point) for the vertical displacement of one point in the railway track, due to the passage of one axle for a train travelling at $200 \mathrm{~km} / \mathrm{h}$ (cf. [7] for more details). A good agreement between the calculated and the measured displacement is achieved and the dynamic effects described in [6] have been correctly modelled.

\section{Correspondence with the case of invariant structures}

At first, it should be noted that using Eqs. (22) and (23), we can deduce the Floquet transform of $\hat{\mathbf{h}}$ with respect to the variable $\mathbf{y}$ from its Floquet transform with respect to the variable $\mathbf{x}$ :

$$
\tilde{\hat{\mathbf{h}}}_{\mathbf{y}}(\mathbf{x}, \tilde{\mathbf{y}}, \kappa)=\tilde{\hat{\mathbf{h}}}_{\mathbf{x}}(\mathbf{x}, \tilde{\mathbf{y}},-\kappa) \quad \text { with } \mathbf{x}=\tilde{\mathbf{x}}
$$

The structure is now assumed to be invariant in the $\mathbf{e}_{2}$-direction. Consider Eq. (19) which is deduced from Eq. (17) without any assumption about the periodicity. Using notations introduced in Eq. (7), it can be rearranged as follows:

$$
\hat{\mathbf{u}}=\frac{1}{2 \pi} \int_{-\infty}^{+\infty} \hat{g}\left(k_{y_{2}}\right) \mathrm{e}^{\mathrm{i} k^{*} X_{2}} \hat{\hat{\mathbf{h}}}_{X_{1}, X_{3}}\left(-k^{*}\right) \mathrm{d} k_{y_{2}}
$$

with

$$
\hat{\hat{\mathbf{h}}}_{X_{1}, X_{3}}\left(-k^{*}\right)=\int_{-\infty}^{+\infty} \mathrm{e}^{-\mathrm{i} k^{*} y_{2}} \hat{\mathbf{h}}\left(y_{2}\right) \mathrm{d} y_{2} \quad \text { and } \quad k^{*}=k_{y_{2}}-\frac{\omega}{V}
$$


Using Eqs. (8) and (27), we deduce that:

$$
\hat{\hat{\mathbf{h}}}_{X_{1}, X_{3}}\left(-k^{*}\right)=\int_{0}^{L} \mathrm{e}^{-\mathrm{i} k^{*} \tilde{y}_{2}} \tilde{\hat{\mathbf{h}}}_{\mathbf{y}}\left(\tilde{y}_{2},-\kappa_{0}\right) \mathrm{d} \tilde{y}_{2}
$$

with $\kappa_{0}=k^{*}-\frac{2 m \pi}{L}$. Replacing $\hat{\hat{\mathbf{h}}}_{X_{1}, X_{3}}$ in Eq. (28) by its expression given in Eq. (30), it is shown that the response of periodic structures to moving loads using Floquet transforms (expressed by Eq. (24)) is equivalent to the response due to moving loads of invariant structures using Fourier transforms (expressed by Eq. (28)).

\section{Conclusions}

In this Note, Floquet transforms are used to obtain the response of periodic structures due to moving loads from the transfer function in the frequency-wavenumber domain calculated in an unbounded generic cell. The proposed method has been applied for a real railway structure. Finally, we established the equivalence of this new solution with the response of invariant structures using Fourier transforms.

\section{Acknowledgements}

The proposed work has been supported by the European Community throughout the projects CONVURT ("Control of Vibration from Underground Rail Traffic"-G3RD-CT00-00381) and SUPERTRACK ("Sustained Performance of Railway Tracks"-G1RD-CT-2002-00777).

\section{References}

[1] L. Frỳba, Vibration of Solids and Structures under Moving Loads, Thomas Telford Ltd., London, 1999.

[2] M.G. Floquet, Sur les équations différentielles linéaires à coefficients périodiques, Ann. Ecole Normale 12 (1883).

[3] L. Jezequel, Analysis of the critical speeds of a moving load on an infinite periodically supported beam, J. Sound Vib. 73 (4) (1980) 606-610.

[4] A.I. Vesnitskii, A.V. Metrikine, Parametric instability in the oscillations of a body moving uniformly in a periodically inhomogeneous elastic system, J. Appl. Mech. Tech. Phys. 34 (2) (1993) 266-271.

[5] D. Clouteau, D. Aubry, M.L. Elhabre, Periodic BEM and FEM-BEM coupling: applications to seismic behaviour of very long structures, Comp. Mech. 25 (6) (2000) 567-577.

[6] A.M. Kaynia, C. Madshus, P. Zackrisson, Ground vibration from high-speed trains: prediction and countermeasure, J. Geotech. Geoenv. Eng. 126 (6) (2000) 531-537.

[7] H. Chebli, D. Clouteau, A. Modaressi, Three-dimensional periodic model for the simulation of vibrations induced by high speed trains, Ital. Geotech. J. 38 (4) (2004) 26-31. 